\title{
Education Workshop
}

E-1

Hands-on RNA-Seq Workshop Using the CyVerse Computational Infrastructure. WILLIAM BARBAZUK. University of Florida, Department of Biology and the UF Genetics Institute, Cancer \& Genetics Research Complex, Gainesville, FL 32610. Email: bbarbazuk@ufl.edu

RNA-Seq is a powerful and easily assessable method to examine and compare gene expression on a broad scale. This workshop will focus on introducing researchers to the principles behind RNA-Seq analysis and will provide a hands-on introduction to software and analysis pipelines for RNA-Seq. The workshop is aimed at teaching the computational process that takes the raw data all the way through the high level analysis. Theory and hands-on application will be centered around two RNA-Seq analysis suites: (1) Cufflinks and Stingtie are expression analysis modules that assemble raw reads into transcripts and then evaluate expression, and (2) DeSeq2 which is an R package that works directly on count data. There is no prerequisite programming experience necessary. Raw and analyzed data will be hosted on CyVerse - an NSF funded life sciences cyber-infrastructure platform - that allows analysis to be performed in a web-based environment. Data analysis and demonstrations will be performed on the CyVerse Discovery Environment (DE). For the best experience, students are requested to establish a free CyVerse account (www.cyverse.org) prior to the workshop and should have access to a networked laptop. 